\title{
L'anthropologie face aux problèmes du monde moderne de Claude LÉVI-STRAUSS
}

\section{Raymond MAYER}

\section{OpenEdition}

\section{Journals}

\section{Édition électronique}

URL : http://journals.openedition.org/jso/6457

DOI : $10.4000 /$ jso. 6457

ISSN : $1760-7256$

\section{Éditeur}

Société des océanistes

\section{Édition imprimée}

Date de publication : 15 décembre 2011

Pagination : 446-448

ISBN : 978-2-85430-037-4

ISSN : 0300-953x

\section{Référence électronique}

Raymond MAYER, "L'anthropologie face aux problèmes du monde moderne de Claude LÉVI-STRAUSS 》, Journal de la Société des Océanistes [En ligne], 133 | 2e semestre 2011, mis en ligne le 31 décembre 2011, consulté le 22 septembre 2020. URL : http://journals.openedition.org/jso/6457 ; DOI : https:// doi.org/10.4000/jso.6457

Ce document a été généré automatiquement le 22 septembre 2020.

(c) Tous droits réservés 


\title{
L'anthropologie face aux problèmes $\mathrm{du}$ monde moderne de Claude LÉVI-
}

\section{STRAUSS}

\author{
Raymond MAYER
}

\section{RÉFÉRENCE}

LÉVI-STRAUSS Claude, 2011. L'anthropologie face aux problèmes du monde moderne, Paris, Seuil, coll. La librairie du xxi siècle, 150 p.

1 Coup sur coup, les éditions du Seuil ont publié, en avril 2011, deux ouvrages posthumes de Claude Lévi-Strauss (voir aussi le compte rendu qui suit). Ceux-ci rassemblent soit des textes inédits, soit des textes parus dans des revues étrangères, principalement japonaises, et donc difficilement accessibles. Le premier livre est un livre de conférences, le second un livre d'articles. Même si les conférences éditées dans le premier livre furent prononcées au cours d'un voyage ancien - précisément en 1986 -, à l'occasion d'une invitation de leur auteur au Japon, leur publication posthume constitue un plaisir de lecture. Ces conférences n'ont sans doute pas valeur testamentaire, comme il est permis de le penser d'un texte plus tardif donné à l'occasion du soixantième anniversaire de la création de l'Unesco (2005). Ces textes peuvent toutefois entrer dans la catégorie de Paroles données, pour reprendre un titre choisi par Lévi-Strauss quand il vint à publier ses cours professés de 1960 à 1982 au Collège de France. Et ils explicitent son point de vue sur des questions éminemment contemporaines, ce qui est plutôt rare dans son œuvre.

2 La première conférence (pp. 11-58) porte le titre « La fin de la suprématie culturelle de l'Occident ». À l'âge de 78 ans, Lévi-Strauss ne prophétise pas, il analyse. Il adopte quasiment une posture postmoderne en déclarant :

«La civilisation de type occidental a perdu le modèle qu'elle s'était donné à elle-

même, et elle n'ose plus offrir ce modèle aux autres. » (p. 16) 
3 Toute la différence entre le $\mathrm{XIX}^{\mathrm{e}}$ siècle conquérant et sûr de son progrès et le $\mathrm{xx}^{\mathrm{e}}$ siècle miné par ses doutes - et même par la certitude que le progrès matériel mine les ressources de la planète - est affichée dans cette phrase. Sans doute n'y a-t-il là qu'une réflexion de sens commun que pourrait emprunter n'importe quel sympathisant d'un mouvement écologique. Mais il s'y ajoute l'affirmation que l'expansion du modèle occidental est définitivement invalidée par son aporie finale, et vouée de ce fait à l'illégitimité.

En 1986, le terme SIDA entre dans le texte de cette conférence :

«La maladie dite en français SIDA, en anglais AIDS, écrit alors Lévi-Strauss, offre un exemple d'actualité. Cette maladie virale, localisée dans quelques foyers d'Afrique tropicale où elle vivait probablement en équilibre avec les populations indigènes depuis des millénaires, est devenue un risque majeur quand les hasards de l'histoire l'ont introduite dans des sociétés plus volumineuses. » (p. 29)

5 On le voit : la conférence est à la fois en prise avec l'actualité médicale de la décennie 1980 en même temps qu'elle maintient la priorité des analyses anthropologiques aux seuls groupes restreints de population.

6 La deuxième conférence (pp. 61-104) porte un titre pour le moins énigmatique : «Trois grands problèmes contemporains: la sexualité, le développement économique et la pensée mythique ». L'appariement des trois thèmes pourrait faire penser à un mélange des genres. Trouver le développement économique (quasi absent dans ses écrits) coincé entre la sexualité et la pensée mythique paraît pour le moins iconoclaste. Mais les propos sont parmi les plus avancés sur des phénomènes de société contemporaine sur lesquels Lévi-Strauss se soit prononcé.

7 Ainsi la procréation artificielle, la virginité et les couples homosexuels font-ils leur apparition dans l'argumentaire de Lévi-Strauss :

«Sur toutes ces questions, les anthropologues ont beaucoup à dire, parce que les sociétés qu'ils étudient se sont posé ces problèmes et qu'elles en offrent des solutions. Bien sûr, ces sociétés ignorent les techniques modernes de fécondation in vitro, de prélèvement d'ovule ou d'embryon, de transfert, d'implantation et de congélation. Mais elles ont imaginé et mis en pratique des formules équivalentes, au moins sous les angles juridique et psychologique. Permettez-moi de donner quelques exemples. » (p. 69)

8 Et Lévi-Strauss de citer en effet l'exemple des Samo du Burkina Faso qui font précéder un mariage d'une période "légale » d'un amant officiel pendant trois ans, et la reconnaissance, par le futur mari, de l'enfant né des œuvres de l'amant.

«De son côté, un homme peut avoir plusieurs épouses légitimes, mais, si elles le quittent, il restera le père légal de tous les enfants qu'elles mettront au monde par la suite. » (p. 70)

Dans d'autres populations africaines, « un homme marié dont la femme est stérile peut [...] moyennant paiement, s'entendre avec une femme féconde. [...] En ce cas, le mari légal est donneur inséminateur, et la femme loue son ventre à un autre homme, ou à un couple sans enfant. La question, brûlante en France, de savoir si la prêteuse d'utérus doit le faire gratuitement ou peut recevoir une rémunération ne se pose donc pas » (p. 70).

«Les sociétés sans écriture connaissent aussi des équivalents de l'insémination post mortem que les tribunaux français interdisent. [...] Et pourtant, une institution attestée depuis des millénaires (car elle existait déjà chez les anciens 
Hébreux), le lévirat, permettait et même parfois imposait que le frère cadet engendre au nom de son frère mort. » (p. 71)

Et Lévi-Strauss d'aligner les exemples où selon lui « toutes ces formules offrent autant d'images métaphoriques anticipées des techniques modernes » (p. 73). La conclusion est très claire :

«L'anthropologue ne propose pas à ses contemporains d'adopter les idées et les coutumes de telle ou telle population exotique » (p. 73)

mais cette négation se double de deux affirmations :

«l'anthropologie révèle que ce que nous considérons comme "naturel" [...] se réduit à des contraintes et à des habitudes mentales propres à notre culture "; « en second lieu, [...] nous pouvons suggérer dans quels cadres se développeront des évolutions encore incertaines, mais qu'on aurait tort de dénoncer par avance comme des déviations ou des perversions. Le grand débat qui se déroule actuellement au sujet de la procréation assistée est de savoir s'il convient de légiférer, sur quoi, et dans quel sens. » (p. 74)

Ce qui est vrai de la procréation artificielle l'est aussi, aux yeux de Lévi-Strauss, de la vie économique.

«Si toute l'activité des sociétés à marché relevait des lois économiques, la science économique serait une science véritable, permettant de prévoir et d'agir, ce qui n'est manifestement pas le cas. On peut voir là la preuve que même dans des conduites qui nous semblent purement économiques, d'autres facteurs interviennent et prennent la science économique en défaut. Mais ces facteurs restent voilés pour nous derrière un écran de prétendue rationalité, et l'étude de sociétés différentes, qui leur donnent plus d'importance, nous aide à les mettre en évidence.» (p. 77)

Et l'auteur de rappeler des exemples d'activités quasi industrielles dans des sociétés réputées primitives, et la perte de principes nutritifs due aux sélections botaniques des systèmes agricoles.

Commentant l'exercice de la pensée mythique,

«on ne doit pas s'étonner si les techniques, les objets manufacturés sont dévalorisés par la pensée indigène chaque fois qu'il s'agit de l'essentiel, c'est-à-dire des relations entre l'homme et le monde surnaturel. » (p. 85)

"Si de misérables communautés indigènes d'Amérique du Nord et d'Australie ont longtemps refusé - refusent toujours dans certains cas - de céder des territoires moyennant des indemnités parfois énormes, c'est, au témoignage même des intéressés, parce qu'ils voient dans le sol ancestral une "mère ". » (p. 86)

"Leur structure interne a une trame plus serrée, un décor plus riche que celle des civilisations complexes. Aussi, des sociétés de très bas niveau technique et économique peuvent éprouver un sentiment de bien-être et de plénitude : chacune estime offrir à ses membres la seule vie qui mérite d'être vécue. » (p. 88)

Il refait alors la démonstration de l'utilité de sa fameuse distinction entre "sociétés chaudes » propices au changement et « sociétés froides » hostiles au changement.

La troisième conférence (pp.105-146) est encore davantage dans l'air du temps et a trouvé sa marque de fabrique auprès de l'UNESCO :

«Reconnaissance de la diversité culturelle: ce que nous apprend la culture japonaise. »

16 Comme dans les deux premières conférences, Lévi-Strauss part d'un point de vue général sur le racisme en se servant des résultats des études génétiques qui commençaient à poindre sur le marché scientifique pour aboutir à une nouvelle formulation des principes qu'il avait retenus lors de la rédaction de ses opuscules Race 
et histoire (1952) et Race et culture (1973) pour l' unEsco. La finale porte sur des applications aux contextes historique et contemporain japonais, mais celles-ci sont reprises en nombre et en qualité plus importants dans l'ouvrage spécifiquement consacré au Japon (voir compte rendu suivant). 